\title{
Forced Oscillation of Nonlinear Impulsive Hyperbolic Partial Differential Equation with Several Delays
}

\author{
Vadivel Sadhasivam, Jayapal Kavitha, Thangaraj Raja \\ Post Graduate and Research Department of Mathematics, Thiruvalluvar Government Arts College, Rasipuram, \\ India \\ Email: ovsadha@gmail.com, kaviakshita@gmail.com,trmaths19@gmail.com
}

Received 17 October 2015; accepted 24 November 2015; published 27 November 2015

Copyright @ 2015 by authors and Scientific Research Publishing Inc.

This work is licensed under the Creative Commons Attribution International License (CC BY). http://creativecommons.org/licenses/by/4.0/

(c) (7) Open Access

\begin{abstract}
In this paper, we study oscillatory properties of solutions for the nonlinear impulsive hyperbolic equations with several delays. We establish sufficient conditions for oscillation of all solutions.
\end{abstract}

\section{Keywords}

\section{Oscillation, Hyperbolic Equation, Impulsive, Delays}

\section{Introduction}

The theory of partial functional differential equations can be applied to many fields, such as biology, population growth, engineering, control theory, physics and chemistry, see the monograph [1] for basic theory and applications. The oscillation of partial functional differential equations has been studied by many authors see, for example [2]-[7], and the references cited therein.

The theory of impulsive partial differential systems makes its beginning with the paper [8] in 1991. In recent years, the investigation of oscillations of impulsive partial differential systems has attracted more and more attention in the literature see, for example [9]-[13]. Recently, the investigation on the oscillations of impulsive partial differential systems with delays can be found in [14]-[19].

To the best of our knowledge, there is little work reported on the oscillation of second order impulsive partial functional differential equation with delays. Motivated by this observation, in this paper we study the oscillation of nonlinear forced impulsive hyperbolic partial differential equation with several delays of the form 


$$
\begin{aligned}
& \frac{\partial}{\partial t}\left[r(t) \frac{\partial}{\partial t}(u(x, t))\right]=a(t) \Delta u(x, t)-p(x, t) f(u(x, t)) \\
& \left.-\sum_{j=1}^{n} q_{j}(x, t) f_{j}\left(u\left(x, t-\sigma_{j}\right)\right)+F(x, t), \quad t \neq t_{k},(x, t) \in \Omega \times \mathbb{R}^{+}=G\right\} \\
& u\left(x, t_{k}^{+}\right)=\alpha_{k}\left(x, t_{k}, u\left(x, t_{k}\right)\right) \text {, } \\
& u_{t}\left(x, t_{k}^{+}\right)=\beta_{k}\left(x, t_{k}, u_{t}\left(x, t_{k}\right)\right), \quad t=t_{k}, \quad k=1,2, \cdots .
\end{aligned}
$$

with the boundary conditions

$$
\begin{gathered}
\frac{\partial u}{\partial \gamma}+h(x) u=g(x, t), \quad(x, t) \in \partial \Omega \times \mathbb{R}^{+} \\
u=\varphi(x, t), \quad(x, t) \in \partial \Omega \times \mathbb{R}^{+}
\end{gathered}
$$

and the initial condition

$$
u(x, t)=\Phi(x, t), \quad(x, t) \in[-\delta, 0] \times \Omega .
$$

Here $\Omega \subset \mathbb{R}^{N}$ is a bounded domain with boundary $\partial \Omega$ smooth enough and $\Delta$ is the Laplacian in the Euclidean $N$-space $\mathbb{R}^{N}, \gamma$ is a unit exterior normal vector of $\partial \Omega, \delta=\max \left\{\sigma_{j}\right\}$, $\Phi(x, t) \in C^{2}([-\delta, 0] \times \Omega, \mathbb{R})$.

In the sequal, we assume that the following conditions are fulfilled:

(H1) $r(t), a(t) \in P C\left(\mathbb{R}^{+}, \mathbb{R}^{+}\right), \sigma_{j}$ is a positive constant, $p(x, t), q_{j}(x, t)$ are class of functions which are piece wise continuous in $t$ with discontinuities of first kind only at $t=t_{k}, k=1,2, \cdots$ and left continuous at $t=t_{k}, k=1,2, \cdots$

(H2) $f(u), f_{j}(u) \in C\left(\mathbb{R}^{+}, \mathbb{R}^{+}\right) ; \frac{f(u)}{u} \geq C$ is a positive constant, $\frac{f_{j}(u)}{u} \geq C_{j}$ is a positive constant, for $u \neq 0 ; \quad h(x) \in C\left(\partial \Omega \times \Omega^{+}, \mathbb{R}\right) ; \quad F(x, t) \in P C\left(\mathbb{R}^{+} \times \bar{\Omega}, \mathbb{R}\right) ; \quad g(t, x) \quad$ and $\quad \varphi(t, x) \in P C\left(\mathbb{R}^{+} \times \partial \Omega, \mathbb{R}\right) ;$ $0<t_{1}<t_{2}<\cdots<t_{k}<\cdots, \lim _{k \rightarrow \infty} t_{k}=\infty$.

(H3) $u(x, t)$ and their derivatives $u_{t}(x, t)$ are piecewise continuous in $t$ with discontinuities of first kind only at $t=t_{k}, k=1,2, \cdots, \quad$ and left continuous at $t=t_{k}, \quad u\left(x, t_{k}\right)=u\left(x, t_{k}^{-}\right), \quad u_{t}\left(x, t_{k}\right)=u_{t}\left(x, t_{k}^{-}\right), \quad k=1,2, \cdots$.

(H4) $\alpha_{k}\left(x, t_{k}, u\left(x, t_{k}\right)\right), \beta_{k}\left(x, t_{k}, u_{t}\left(x, t_{k}\right)\right) \in P C\left(\mathbb{R}^{+} \times \bar{\Omega} \times \mathbb{R}, \mathbb{R}\right), \quad k=1,2, \cdots$, and there exist positive constants $a_{k}, a_{k}^{*}, b_{k}, b_{k}^{*}$ and $b_{k} \leq a_{k}^{*}$ such that for $k=1,2, \cdots$,

$$
\begin{aligned}
& a_{k}^{*} \leq \frac{\alpha_{k}\left(x, t_{k}, \xi\right)}{\xi} \leq a_{k} \\
& b_{k}^{*} \leq \frac{\beta_{k}\left(x, t_{k}, \xi\right)}{\xi} \leq b_{k} .
\end{aligned}
$$

Let us construct the sequence $\left\{\bar{t}_{k}\right\}=\left\{t_{k}\right\} \bigcup\left\{t_{k \sigma_{j}}\right\}$, where $t_{k \sigma_{j}}=t_{k}+\sigma_{j}$ and $\bar{t}_{k}<\bar{t}_{k+1}, \quad k=1,2, \cdots$.

By a solution of problem (1), (2) ((1),(3)) with initial condition (4), we mean that any function $u(x, t)$ for which the following conditions are valid:

1. If $-\delta \leq t \leq 0$, then $u(x, t)=\Phi(x, t)$.

2. If $0 \leq t \leq \bar{t}_{1}=t_{1}$, then $u(x, t)$ coincides with the solution of the problem (1) and (2) ((3)) with initial condition.

3. If $\bar{t}_{k}<t \leq \bar{t}_{k+1}, \bar{t}_{k} \in\left\{t_{k}\right\} \backslash\left\{t_{k \sigma_{j}}\right\}$, then $u(x, t)$ coincides with the solution of the problem (1) and (2) ((3)). 
4. If $\bar{t}_{k}<t \leq \bar{t}_{k+1}, \bar{t}_{k} \in\left\{t_{k \sigma_{j}}\right\}$, then $u(x, t)$ coincides with the solution of the problem (2) ((3)) and the following equations

$$
\begin{aligned}
& \frac{\partial}{\partial t}\left[r(t) \frac{\partial}{\partial t}\left(u\left(x, t^{+}\right)\right)\right]=a(t) \Delta u\left(x, t^{+}\right)-p(x, t) f\left(u\left(x, t^{+}\right)\right) \\
& -\sum_{j=1}^{n} q_{j}(x, t) f_{j}\left(u\left(x,\left(t-\sigma_{j}\right)^{+}\right)\right)+F(x, t), \quad t \neq t_{k}, \quad(x, t) \in \Omega \times \mathbb{R}^{+}=G \\
& u\left(x, \bar{t}_{k}^{+}\right)=u\left(x, \bar{t}_{k}\right), \quad u_{t}\left(x, \bar{t}_{k}^{+}\right)=u_{t}\left(x, \bar{t}_{k}\right), \quad \text { for } \bar{t}_{k} \in\left\{t_{k}\right\} \backslash\left\{t_{k \sigma_{j}}\right\},
\end{aligned}
$$

or

$$
u\left(x, \bar{t}_{k}^{+}\right)=\alpha_{k_{i}}\left(x, \bar{t}_{k}, u\left(x, \bar{t}_{k}\right)\right), \quad u_{t}\left(x, \bar{t}_{k}^{+}\right)=\beta_{k_{i}}\left(x, \bar{t}_{k}, u_{t}\left(x, \bar{t}_{k}\right)\right), \quad \text { for } \bar{t}_{k} \in\left\{t_{k \sigma_{j}}\right\} \cap\left\{t_{k}\right\} .
$$

Here the number $k_{i}$ is determined by the equality $\bar{t}_{k}=t_{k_{i}}$.

We introduce the notations:

$$
\begin{aligned}
& \Gamma_{k}=\left\{(x, t): t \in\left(t_{k}, t_{k+1}\right), x \in \Omega\right\} ; \quad \Gamma=\bigcup_{k=0}^{\infty} \Gamma_{k}, \\
& \bar{\Gamma}_{k}=\left\{(x, t): t \in\left(t_{k}, t_{k+1}\right), x \in \bar{\Omega}\right\} ; \quad \bar{\Gamma}=\bigcup_{k=0}^{\infty} \bar{\Gamma}_{k}, \\
& p(t)=\min _{x \in \bar{\Omega}} p(x, t) \quad \text { and } \quad q_{j}(t)=\min _{x \in \bar{\Omega}} q_{j}(x, t) .
\end{aligned}
$$

The solution $u \in C^{2}(\Gamma) \cap C^{1}(\bar{\Gamma})$ of problem (1), (2) ((1),(3)) is called nonoscillatory in the domain $\mathrm{G}$ if it is either eventually positive or eventually negative. Otherwise, it is called oscillatory.

This paper is organized as follows: Section 2, deals with the oscillatory properties of solutions for the problem (1) and (2). In Section 3, we discuss the oscillatory properties of solutions for the problem (1) and (3). Section 4 presents some examples to illustrate the main results.

\section{Oscillation Properties of the Problem (1) and (2)}

To prove the main result, we need the following lemmas.

Lemma 2.1. Suppose that $\lambda$ is the minimum positive eigenvalue of the problem

$$
\begin{gathered}
\Delta \eta(x)+\lambda \eta(x)=0, \quad x \in \Omega, \\
\frac{\partial \eta}{\partial \gamma}+h(x) \eta(x)=0, \quad x \in \partial \Omega,
\end{gathered}
$$

and $\eta(x)$ is the corresponding eigenfunction of $\lambda$. Then $\lambda>0$ and $\eta(x)>0, x \in \Omega$. Proof. The proof of the lemma can be found in [20].

Lemma 2.2. Let $u(x, t) \in C^{2}(\Gamma) \cap C^{1}(\bar{\Gamma})$ be a positive solution of the problem (1), (2) in $G$. Then the functions

$$
v(t)=\int_{\Omega} u(x, t) \eta(x) \mathrm{d} x \quad \text { and } \quad \int_{\Omega} F(x, t) \eta(x) \mathrm{d} x \leq 0
$$

are satisfies the impulsive differential inequality

$$
\begin{gathered}
{\left[r(t) v^{\prime}(t)\right]^{\prime}+\lambda a(t) v(t)+C p(t) v(t)+\sum_{j=1}^{n} C_{j} q_{j}(t) v\left(t-\sigma_{j}\right) \leq R(t), \quad t \neq t_{k}} \\
a_{k}^{*} \leq \frac{v\left(t_{k}^{+}\right)}{v\left(t_{k}\right)} \leq a_{k} \\
b_{k}^{*} \leq \frac{v^{\prime}\left(t_{k}^{+}\right)}{v^{\prime}\left(t_{k}\right)} \leq b_{k}, \quad k=1,2, \cdots
\end{gathered}
$$


where

$$
R(t)=a(t) \int_{\partial \Omega} \eta(x) g(x, t) \mathrm{d} S .
$$

has an eventually positive solution.

Proof. Let $u(x, t)$ be a positive solution of the problem (1), (2) in $G$. Without loss of generality, we may assume that there exists a $T>0, t_{0}>T$ such that $u(x, t)>0, u\left(x, t-\sigma_{j}\right)>0, j=1,2, \cdots, n$, for

$$
(x, t) \in \Omega \times\left[t_{0}, \infty\right) .
$$

For $t \geq t_{0}, t \neq t_{k}, k=1,2, \cdots$, multiplying Equation (1) with $\eta(x)$, which is the same as that in Lemma 2.1 and then integrating (1) with respect to $x$ over $\Omega$ yields

$$
\begin{aligned}
\frac{\mathrm{d}}{\mathrm{d} t}\left[r(t) \frac{\mathrm{d}}{\mathrm{d} t} \int_{\Omega} u(x, t) \eta(x) \mathrm{d} x\right]= & a(t) \int_{\Omega} \Delta u(x, t) \eta(x) \mathrm{d} x-\int_{\Omega} p(x, t) f(u(x, t)) \eta(x) \mathrm{d} x \\
& -\sum_{j=1}^{n} \int_{\Omega} q_{j}(x, t) f_{j}\left(u\left(x, t-\sigma_{j}\right)\right) \eta(x) \mathrm{d} x+\int_{\Omega} F(x, t) \eta(x) \mathrm{d} x .
\end{aligned}
$$

By Green's formula, and the boundary condition we have

$$
\begin{aligned}
\int_{\Omega} \Delta u(x, t) \eta(x) \mathrm{d} x & =\int_{\partial \Omega}\left(\eta \frac{\partial u}{\partial \gamma}-u \frac{\partial \eta}{\partial \gamma}\right) \mathrm{d} S+\int_{\Omega} u \Delta \eta \mathrm{d} x=\int_{\partial \Omega}(\eta(g-h u)-u(-h \eta)) \mathrm{d} S+\int_{\Omega} u(-\lambda \eta) \mathrm{d} x \\
& =\int_{\partial \Omega} \eta(x) g(x, t) \mathrm{d} S-\lambda \int_{\Omega} u(x, t) \eta(x) \mathrm{d} x,
\end{aligned}
$$

where $\mathrm{d} S$ is the surface element on $\partial \Omega$.

Also from condition (H2), and Jenson's inequality we can easily obtain

$$
\begin{gathered}
\int_{\Omega} p(x, t) f(u(x, t)) \eta(x) \mathrm{d} x \geq C p(t) \int_{\Omega} u(x, t) \eta(x) \mathrm{d} x \\
\int_{\Omega} q_{j}(x, t) f_{j}\left(u\left(x, t-\sigma_{j}\right)\right) \eta(x) \mathrm{d} x \geq C_{j} q_{j}(t) \int_{\Omega} u\left(x, t-\sigma_{j}\right) \eta(x) \mathrm{d} x
\end{gathered}
$$

Thus, $v(t)>0$. Hence we obtain the following differential inequality

$$
\begin{aligned}
& \frac{\mathrm{d}}{\mathrm{d} t}\left[r(t) \frac{\mathrm{d}}{\mathrm{d} t} \int_{\Omega} u(x, t) \eta(x) \mathrm{d} x\right]+a(t) \lambda \int_{\Omega} u(x, t) \eta(x) \mathrm{d} x+C p(t) \int_{\Omega} u(x, t) \eta(x) \mathrm{d} x \\
& -\sum_{j=1}^{n} C_{j} q_{j}(t) \int_{\Omega} u\left(x, t-\sigma_{j}\right) \eta(x) \mathrm{d} x \leq a(t) \int_{\partial \Omega} g(x, t) \eta(x) \mathrm{d} S, \\
& \quad\left[r(t) v^{\prime}(t)\right]^{\prime}+\lambda a(t) v(t)+C p(t) v(t)+\sum_{j=1}^{n} C_{j} q_{j}(t) v\left(t-\sigma_{j}\right) \leq R(t), t \neq t_{k}
\end{aligned}
$$

where

$$
R(t)=a(t) \int_{\partial \Omega} \eta(x) g(x, t) \mathrm{d} S .
$$

For $t \geq t_{0}, t=t_{k}, k=1,2, \cdots$, from (1) and condition (H4), we obtain

$$
\begin{aligned}
& a_{k}^{*} \leq \frac{u\left(x, t_{k}^{+}\right)}{u\left(x, t_{k}\right)} \leq a_{k} \\
& b_{k}^{*} \leq \frac{u_{t}\left(x, t_{k}^{+}\right)}{u_{t}\left(x, t_{k}\right)} \leq b_{k} .
\end{aligned}
$$

According to $v(t)=\int_{\Omega} u(x, t) \eta(x) \mathrm{d} x$, we obtain 


$$
\begin{gathered}
a_{k}^{*} \leq \frac{v\left(t_{k}^{+}\right)}{v\left(t_{k}\right)} \leq a_{k} \\
b_{k}^{*} \leq \frac{v^{\prime}\left(t_{k}^{+}\right)}{v^{\prime}\left(t_{k}\right)} \leq b_{k}, \quad k=1,2, \cdots .
\end{gathered}
$$

Hence, we obtain that $v(t)>0$ is a positive solution of impulsive differential inequalities (5)-(7).

This completes the proof.

Lemma 2.3. Let $u(x, t) \in C^{2}(\Gamma) \cap C^{1}(\bar{\Gamma})$ be a positive solution of the problem (1), (2) in $G$. If we further assume that $f(-u)=-f(u), \quad u \in(0,+\infty)$ and the impulsive differential inequality (5), and

$$
\begin{gathered}
{\left[r(t) v^{\prime}(t)\right]^{\prime}+\lambda a(t) v(t)+C p(t) v(t)+\sum_{j=1}^{n} C_{j} q_{j}(t) v\left(t-\sigma_{j}\right) \leq-R(t), t \neq t_{k}} \\
a_{k}^{*} \leq \frac{v\left(t_{k}^{+}\right)}{v\left(t_{k}\right)} \leq a_{k} \\
b_{k}^{*} \leq \frac{v^{\prime}\left(t_{k}^{+}\right)}{v^{\prime}\left(t_{k}\right)} \leq b_{k}, \quad k=1,2, \cdots
\end{gathered}
$$

have no eventually positive solution, then each nonzero solution of the problem (1)-(2) is oscillatory in the domain $G$.

Proof. Let $u(x, t)$ be a positive solution of the problem (1), (2) in G. Without loss of generality, we may assume that there exists a $T>0, t_{0}>T$ such that $u(x, t)>0, u\left(x, t-\sigma_{j}\right)>0, j=1,2, \cdots, n$, for $(x, t) \in \Omega \times\left[t_{0}, \infty\right)$.

From Lemma 2.2, it follows that the function $v(t)$ is an eventually positive solution of the inequality (5) which is a contradictions.

If $u(x, t)<0$ for $(x, t) \in \Omega \times\left[t_{0}, \infty\right)$, then the function

$$
\tilde{u}(x, t)=-u(x, t), \quad(x, t) \in \Omega \times\left[t_{0}, \infty\right),
$$

is a positive solution of the following impulsive hyperbolic equation

$$
\begin{gathered}
\frac{\partial}{\partial t}\left[r(t) \frac{\partial}{\partial t}(u(x, t))\right]=a(t) \Delta u(x, t)-p(x, t) f(u(x, t)) \\
-\sum_{j=1}^{n} q_{j}(x, t) f_{j}\left(u\left(x, t-\sigma_{j}\right)\right)+F(x, t), \quad t \neq t_{k}, \quad(x, t) \in \Omega \times \mathbb{R}^{+}=G \\
u\left(x, t_{k}^{+}\right)=\alpha_{k}\left(x, t_{k}, u\left(x, t_{k}\right)\right), \\
u_{t}\left(x, t_{k}^{+}\right)=\beta_{k}\left(x, t_{k}, u_{t}\left(x, t_{k}\right)\right), \quad t=t_{k}, \quad k=1,2, \cdots \\
\frac{\partial u}{\partial \gamma}+h(x) u=-g(x, t), \quad(x, t) \in \partial \Omega \times \mathbb{R}^{+}
\end{gathered}
$$

and satisfies

$$
\begin{aligned}
& \frac{\mathrm{d}}{\mathrm{d} t}\left[r(t) \frac{\mathrm{d}}{\mathrm{d} t} \int_{\Omega} \tilde{u}(x, t) \eta(x) \mathrm{d} x\right]+\lambda a(t) \int_{\Omega} \tilde{u}(x, t) \eta(x) \mathrm{d} x+C p(t) \int_{\Omega} \tilde{u}(x, t) \eta(x) \mathrm{d} x \\
& -\sum_{j=1}^{n} C_{j} q_{j}(t) \int_{\Omega} \tilde{u}\left(x, t-\sigma_{j}\right) \eta(x) \mathrm{d} x \leq-a(t) \int_{\partial \Omega} g(x, t) \eta(x) \mathrm{d} S, \\
& {\left[r(t) v^{\prime}(t)\right]^{\prime}+\lambda a(t) v(t)+C p(t) v(t)+\sum_{j=1}^{n} C_{j} q_{j}(t) v\left(t-\sigma_{j}\right) \leq-R(t), t \neq t_{k}}
\end{aligned}
$$


where

$$
R(t)=a(t) \int_{\partial \Omega} \eta(x) g(x, t) \mathrm{d} S .
$$

For $t \geq t_{0}, t=t_{k}, k=1,2, \cdots$, from (1) and condition (H4), we obtain

$$
\begin{aligned}
& a_{k}^{*} \leq \frac{\tilde{u}\left(x, t_{k}^{+}\right)}{\tilde{u}\left(x, t_{k}\right)} \leq a_{k} \\
& b_{k}^{*} \leq \frac{\tilde{u}_{t}\left(x, t_{k}^{+}\right)}{\tilde{u}_{t}\left(x, t_{k}\right)} \leq b_{k} .
\end{aligned}
$$

According to $\tilde{v}(t)=\int_{\Omega} \tilde{u}(x, t) \eta(x) \mathrm{d} x$, we obtain

$$
\begin{gathered}
a_{k}^{*} \leq \frac{v\left(t_{k}^{+}\right)}{v\left(t_{k}\right)} \leq a_{k} \\
b_{k}^{*} \leq \frac{v^{\prime}\left(t_{k}^{+}\right)}{v^{\prime}\left(t_{k}\right)} \leq b_{k}, \quad k=1,2, \cdots .
\end{gathered}
$$

Thus, it follows that the function $\tilde{v}(t)=\int_{\Omega} \tilde{u}(x, t) \eta(x) \mathrm{d} x$ is a positive solution of the inequality (8)-(10) for $t_{0}>T$ which is also a contradiction. This completes the proof.

Now, if we set $g \equiv 0$ in the proof of Lemma 2.3, then we can obtain the following lemma.

Lemma 2.4. Let $u(x, t) \in C^{2}(\Gamma) \cap C^{1}(\bar{\Gamma})$ be a positive solution of the problem (1), (2) in $G$. If we further assume that $f(-u)=-f(u), u \in(0,+\infty)$ and the impulsive differential inequality (5), and

$$
\begin{gathered}
{\left[r(t) v^{\prime}(t)\right]^{\prime}+\lambda a(t) v(t)+C p(t) v(t)} \\
+\sum_{j=1}^{n} C_{j} q_{j}(t) v\left(t-\sigma_{j}\right) \leq 0, \quad t \neq t_{k} \\
a_{k}^{*} \leq \frac{v\left(t_{k}^{+}\right)}{v\left(t_{k}\right)} \leq a_{k} \\
b_{k}^{*} \leq \frac{v^{\prime}\left(t_{k}^{+}\right)}{v^{\prime}\left(t_{k}\right)} \leq b_{k}, \quad k=1,2, \cdots
\end{gathered}
$$

has no eventually positive solution, then each nonzero solution of the problem (1), satisfying the boundary condition

$$
\frac{\partial u}{\partial \gamma}+h(x) u=0, \quad(x, t) \in \partial \Omega \times \mathbb{R}^{+}, t \neq t_{k}
$$

is oscillatory in the domain $G$.

Proof. Let $u(x, t)$ be a positive solution of the problem (1), (2) in G. Without loss of generality, we may assume that there exists a $T>0, t_{0}>T$ such that $u(x, t)>0, u\left(x, t-\sigma_{j}\right)>0, j=1,2, \cdots, n$, for

$$
(x, t) \in \Omega \times\left[t_{0}, \infty\right) .
$$

From Lemma 2.2, it follows that the function $v(t)$ is an eventually positive solution of the inequality (5) which is a contradiction.

If $u(x, t)<0$ for $(x, t) \in \Omega \times\left[t_{0}, \infty\right)$, then the function $\tilde{u}(x, t)=-u(x, t),(x, t) \in \Omega \times\left[t_{0}, \infty\right)$, is a positive solution of the following impulsive hyperbolic equation 


$$
\begin{gathered}
\frac{\partial}{\partial t}\left[r(t) \frac{\partial}{\partial t}(u(x, t))\right]=a(t) \Delta u(x, t)-p(x, t) f(u(x, t)) \\
-\sum_{j=1}^{n} q_{j}(x, t) f_{j}\left(u\left(x, t-\sigma_{j}\right)\right)+f(x, t), \quad t \neq t_{k}, \quad(x, t) \in \Omega \times \mathbb{R}^{+}=G \\
u\left(x, t_{k}^{+}\right)=\alpha_{k}\left(x, t_{k}, u\left(x, t_{k}\right)\right), \\
u_{t}\left(x, t_{k}^{+}\right)=\beta_{k}\left(x, t_{k}, u_{t}\left(x, t_{k}\right)\right), \quad t=t_{k}, \quad k=1,2, \cdots \\
\frac{\partial u}{\partial \gamma}+h(x) u=0, \quad(x, t) \in \partial \Omega \times \mathbb{R}^{+}
\end{gathered}
$$

and satisfies

$$
\begin{aligned}
& \frac{\mathrm{d}}{\mathrm{d} t}\left[r(t) \frac{\mathrm{d}}{\mathrm{d} t} \int_{\Omega} \tilde{u}(x, t) \eta(x) \mathrm{d} x\right]+\lambda a(t) \int_{\Omega} \tilde{u}(x, t) \eta(x) \mathrm{d} x+C p(t) \int_{\Omega} \tilde{u}(x, t) \eta(x) \mathrm{d} x \\
& -\sum_{j=1}^{n} C_{j} q_{j}(t) \int_{\Omega} \tilde{u}\left(x, t-\sigma_{j}\right) \eta(x) \mathrm{d} x \leq 0, \\
& \quad\left[r(t) v^{\prime}(t)\right]^{\prime}+\lambda a(t) v(t)+C p(t) v(t)+\sum_{j=1}^{n} C_{j} q_{j}(t) v\left(t-\sigma_{j}\right) \leq 0, t \neq t_{k} .
\end{aligned}
$$

For $t \geq t_{0}, t=t_{k}, k=1,2, \cdots$, from (1) and condition (H4), we obtain

$$
\begin{gathered}
a_{k}^{*} \leq \frac{\tilde{u}\left(x, t_{k}^{+}\right)}{\tilde{u}\left(x, t_{k}\right)} \leq a_{k} \\
b_{k}^{*} \leq \frac{\tilde{u}_{t}\left(x, t_{k}^{+}\right)}{\tilde{u}_{t}\left(x, t_{k}\right)} \leq b_{k}, \quad k=1,2, \cdots .
\end{gathered}
$$

According to $\tilde{v}(t)=\int_{\Omega} \tilde{u}(x, t) \eta(x) \mathrm{d} x$, we obtain

$$
\begin{aligned}
& a_{k}^{*} \leq \frac{v\left(t_{k}^{*}\right)}{v\left(t_{k}\right)} \leq a_{k} \\
& b_{k}^{*} \leq \frac{v^{\prime}\left(t_{k}^{*}\right)}{v^{\prime}\left(t_{k}\right)} \leq b_{k} .
\end{aligned}
$$

Thus it follows that the function $\tilde{v}(t)=\int_{\Omega} \tilde{u}(x, t) \eta(x) \mathrm{d} x$, is a positive solution of the inequality (11)-(13) for $t_{0}>T$ which is also a contradiction. This completes the proof.

Lemma 2.5. Assume that

(A1) the sequence $\left\{t_{k}\right\}$ satisfies $0<t_{0}<t_{1}<\cdots, \quad \lim _{k \rightarrow \infty} t_{k}=\infty$;

(A2) $m(t) \in P C^{1}\left[\mathbb{R}^{+}, \mathbb{R}\right]$ is left continuous at $t_{k}$ for $k=1,2, \cdots$;

(A3) for $k=1,2, \cdots$ and $t \geq t_{0}$,

$$
\begin{gathered}
m^{\prime}(t) \leq p(t) m(t)+q(t), \quad t \neq t_{k}, \\
m\left(t_{k}^{+}\right) \leq d_{k} m\left(t_{k}\right)+e_{k},
\end{gathered}
$$

where $p(t), q(t) \in C\left(\mathbb{R}^{+}, \mathbb{R}\right), d_{k} \geq 0$ and $e_{k}$ are constants. PC denote the class of piecewise continuous function from $\mathbb{R}^{+}$to $\mathbb{R}$, with discontinuities of the first kind only at $t=t_{k}, k=1,2, \cdots$.

Then 


$$
\begin{aligned}
m(t) \leq & m\left(t_{0}\right) \prod_{t_{0}<t_{k}<t} d_{k} \exp \left(\int_{t_{0}}^{t} p(s) \mathrm{d} s\right)+\int_{t_{0}}^{t} \prod_{s<t_{k}<t} d_{k} \exp \left(\int_{s}^{t} p(r) \mathrm{d} r\right) q(s) \mathrm{d} s \\
& +\sum_{t_{0}<t_{k}<t_{t_{k}<t_{j}<t}} d_{j} \exp \left(\int_{t_{k}}^{t} p(s) \mathrm{d} s\right) e_{k} .
\end{aligned}
$$

Proof. The proof of the lemma can be found in [21].

Lemma 2.6. Let $v(t)$ be an eventually positive (negative) solution of the differential inequality (11)-(13).

Assume that there exists $T \geq t_{0}$ such that $v(t)>0 \quad(v(t)<0)$ for $t \geq T$. If

$$
\lim _{t \rightarrow+\infty} \int_{t_{0}}^{t} \prod_{t_{0}<t_{k}<s} \frac{b_{k}^{*}}{a_{k}} \mathrm{~d} s=+\infty
$$

hold, then $v^{\prime}(t) \geq 0 \quad\left(v^{\prime}(t) \leq 0\right)$ for $t \in\left[T, t_{1}\right] \cup\left(\bigcup_{k=l}^{+\infty}\left(t_{k}, t_{k+1}\right]\right)$, where $l=\min \left\{k: t_{k} \geq T\right\}$.

Proof. The proof of the lemma can be found in [22].

We begin with the following theorem.

Theorem 2.1. If condition (14), and the following condition

$$
\lim _{t \rightarrow+\infty} \int_{t_{0}}^{t} \prod_{t_{0}<t_{k}<s} \frac{a_{k}^{*}}{b_{k}} r\left(t_{k}\right) F(s) \mathrm{d} s=+\infty,
$$

hold, where

$$
F(t)=\frac{\lambda a(t)+C p(t)+\exp \left(-\delta w\left(t_{1}\right)\right) \sum_{j=1}^{n} C_{j} q_{j}(t)}{r(t)},
$$

then every solution of the problem (1), (2) oscillates in G.

Proof. Let $u(x, t)$ be a nonoscillatory solution of (1), (2). Without loss of generality, we can assume that there exists $T>0, t_{0} \geq T$, such that $u(x, t)>0, \quad u\left(x, t-\sigma_{j}\right)>0, \quad j=1,2, \cdots, n$ for $(x, t) \in \Omega \times\left[t_{0}, \infty\right)$.

From Lemma 2.4, we know that $v(t)$ is a positive solution of (11)-(13). Thus from Lemma 2.6, we can find that $v^{\prime}(t) \geq 0$ for $t \geq t_{0}$.

For $t \geq t_{0}, \quad t \neq t_{k}, \quad k=1,2, \cdots$, define

$$
w(t)=r(t) \frac{v^{\prime}(t)}{v(t)}, \quad t \geq t_{0} .
$$

Then we have $w(t)>0, \quad t \geq t_{0}, \quad r(t) v^{\prime}(t)-w(t) v(t)=0$. We may assume that $v\left(t_{0}\right)=1$, thus we have that for $t \geq t_{0}$

$$
\begin{gathered}
v(t)=\exp \left(\int_{t_{0}}^{t} w(s) \mathrm{d} s\right), \\
v^{\prime}(t)=w(t) \exp \left(\int_{t_{0}}^{t} w(s) \mathrm{d} s\right), \\
v^{\prime \prime}(t)=w^{2}(t) \exp \left(\int_{t_{0}}^{t} w(s) \mathrm{d} s\right)+w^{\prime}(t) \exp \left(\int_{t_{0}}^{t} w(s) \mathrm{d} s\right) .
\end{gathered}
$$

Substitute (16)-(18) into (11) and then we obtain,

$$
\begin{aligned}
& r^{\prime}(t) w(t) \exp \left(\int_{t_{0}}^{t} w(s) \mathrm{d} s\right)+r(t)\left[w^{2}(t) \exp \left(\int_{t_{0}}^{t} w(s) \mathrm{d} s\right)+w^{\prime}(t) \exp \left(\int_{t_{0}}^{t} w(s) \mathrm{d} s\right)\right] \\
& +\lambda a(t) \exp \left(\int_{t_{0}}^{t} w(s) \mathrm{d} s\right)+C p(t) \exp \left(\int_{t_{0}}^{t} w(s) \mathrm{d} s\right)+\sum_{j=1}^{n} C_{j} q_{j}(t) \exp \left(\int_{t_{0}}^{t-\sigma_{j}} w(s) \mathrm{d} s\right) \leq 0 .
\end{aligned}
$$

Hence we have 


$$
r(t) w^{2}(t)+r(t) w^{\prime}(t)+\lambda a(t)+C p(t)+\sum_{j=1}^{n} C_{j} q_{j}(t) \exp \left(-\int_{t-\sigma_{j}}^{t} w(s) \mathrm{d} s\right) \leq 0, \quad t \neq t_{k},
$$

or

$$
r(t) w^{\prime}(t)+\lambda a(t)+C p(t)+\sum_{j=1}^{n} C_{j} q_{j}(t) \exp \left(-\int_{t-\sigma_{j}}^{t} w(s) \mathrm{d} s\right) \leq 0, \quad t \neq t_{k} .
$$

From above inequality and condition $b_{k} \leq a_{k}^{*}$, it is easy to see that the function $w(t)$ is nonincreasing for $t \geq t_{1} \geq \delta+t_{0}$. Thus $w(t) \leq w\left(t_{1}\right)$ for $t \geq t_{1}$ which implies that

$$
r(t) w^{\prime}(t)+\lambda a(t)+C p(t)+\exp \left(-\delta w\left(t_{1}\right)\right) \sum_{j=1}^{n} C_{j} q_{j}(t) \leq 0, \quad t \neq t_{k} .
$$

From (12)-(13), we obtain

$$
w\left(t_{k}^{+}\right)=r\left(t_{k}^{+}\right) \frac{v^{\prime}\left(t_{k}^{+}\right)}{v\left(t_{k}^{+}\right)} \leq r\left(t_{k}^{+}\right) \frac{b_{k} v^{\prime}\left(t_{k}\right)}{a_{k}^{*} v\left(t_{k}\right)}=r\left(t_{k}\right) \frac{b_{k}}{a_{k}^{*}} w\left(t_{k}\right),
$$

and

$$
\begin{gathered}
r(t) w^{\prime}(t) \leq-\lambda a(t)-C p(t)-\exp \left(-\delta w\left(t_{1}\right)\right) \sum_{j=1}^{n} C_{j} q_{j}(t), \quad t \neq t_{k} . \\
w\left(t_{k}^{+}\right) \leq r\left(t_{k}\right) \frac{b_{k}}{a_{k}^{*}} w\left(t_{k}\right), \quad k=1,2, \cdots
\end{gathered}
$$

Let

$$
-F(t)=\frac{-\lambda a(t)-C p(t)-\exp \left(-\delta w\left(t_{1}\right)\right) \sum_{j=1}^{n} C_{j} q_{j}(t)}{r(t)} .
$$

Then according to Lemma 2.5, we have

$$
\begin{aligned}
w(t) & \leq w\left(t_{0}\right) \prod_{t_{0}<t_{k}<t} r\left(t_{k}\right) \frac{b_{k}}{a_{k}^{*}}+\int_{t_{0}<t_{k}<t}^{t} r\left(t_{k}\right) \frac{b_{k}}{a_{k}^{*}} F(s) \mathrm{d} s \\
& =\prod_{t_{0}<t_{k}<t} \frac{b_{k}}{a_{k}^{*}}\left[w\left(t_{0}\right) r\left(t_{k}\right)-\int_{t_{t_{0}}<t_{k}<s}^{t} \prod_{k} r\left(t_{k}\right) \frac{a_{k}^{*}}{b_{k}} F(s) \mathrm{d} s\right]<0 .
\end{aligned}
$$

Since $w(t) \geq 0$, the last inequality contradicts condition (15). This completes the proof.

\section{Oscillation Properties of the Problem (1) and (3)}

Next we consider the problem (1) and (3). To prove our main result we need the following lemmas.

Lemma 3.1. Suppose that $\lambda_{0}$ is the smallest positive eigen value of the problem

$$
\begin{array}{ll}
\Delta \Psi(x)+\lambda_{0} \Psi(x)=0, & x \in \Omega, \\
\Psi(x)=0, & x \in \partial \Omega,
\end{array}
$$

and $\Psi(x)$ is the corresponding eigen function of $\lambda_{0}$. Then $\lambda_{0}>0$ and $\Psi(x)>0, x \in \Omega$.

Proof. The proof of the lemma can be found in [20].

Lemma 3.2. Let $u(x, t) \in C^{2}(\Gamma) \cap C^{1}(\bar{\Gamma})$ be a positive solution of the problem (1), (3) in $G$. Then the function

$$
\int_{\Omega} F(x, t) \Psi(x) \mathrm{d} x \leq 0
$$

are satisfies the impulsive differential inequality 


$$
\begin{gathered}
{\left[r(t) v^{\prime}(t)\right]^{\prime}+\lambda_{0} a(t) v(t)+C p(t) v(t)} \\
+\sum_{j=1}^{n} C_{j} q_{j}(t) v\left(t-\sigma_{j}\right) \leq Q(t), t \neq t_{k} \\
a_{k}^{*} \leq \frac{v\left(t_{k}^{+}\right)}{v\left(t_{k}\right)} \leq a_{k} \\
b_{k}^{*} \leq \frac{v^{\prime}\left(t_{k}^{+}\right)}{v^{\prime}\left(t_{k}\right)} \leq b_{k}, \quad k=1,2, \cdots
\end{gathered}
$$

where

$$
Q(t)=-a(t) \int_{\partial \Omega} \varphi(x, t) \frac{\partial \Psi}{\partial N} \mathrm{~d} S
$$

has the eventually positive solution

$$
v(t)=\int_{\Omega} u(x, t) \Psi(x) \mathrm{d} x .
$$

Proof. Let $u(x, t)$ be a positive solution of the problem (1), (3) in G. Without loss of generality, we may assume that there exists a $T>0, t_{0}>T$ such that $u(x, t)>0, u\left(x, t-\sigma_{j}\right)>0, j=1,2, \cdots, n$, for

$$
(x, t) \in \Omega \times\left[t_{0}, \infty\right) .
$$

For $t \geq t_{0}, t \neq t_{k}, k=1,2, \cdots$, multiplying equation (1) with $\Psi(x)$, which is the same as that in Lemma 3.1 and then integrating (1) with respect to $x$ over $\Omega$ yields

$$
\begin{aligned}
\frac{\mathrm{d}}{\mathrm{d} t}\left[r(t) \frac{\mathrm{d}}{\mathrm{d} t} \int_{\Omega} u(x, t) \Psi(x) \mathrm{d} x\right]= & a(t) \int_{\Omega} \Delta u(x, t) \Psi(x) \mathrm{d} x-\int_{\Omega} p(x, t) f(u(x, t)) \Psi(x) \mathrm{d} x \\
& -\sum_{j=1}^{n} \int_{\Omega} q_{j}(x, t) f_{j}\left(u\left(x, t-\sigma_{j}\right)\right) \Psi(x) \mathrm{d} x+\int_{\Omega} F(x, t) \Psi(x) \mathrm{d} x .
\end{aligned}
$$

By Green's formula, and the boundary condition we have

$$
\begin{aligned}
\int_{\Omega} \Delta u(x, t) \Psi(x) \mathrm{d} x & =\int_{\partial \Omega}\left(\Psi \frac{\partial u}{\partial \gamma}-u \frac{\partial \Psi}{\partial \gamma}\right) \mathrm{d} S+\int_{\Omega} u \Delta \Psi \mathrm{d} x \\
& =\int_{\partial \Omega}-\varphi(x, t) \frac{\partial \Psi}{\partial \gamma} \mathrm{d} S+\int_{\Omega} u\left(-\lambda_{0} \Psi\right) \mathrm{d} x \\
& =-\int_{\partial \Omega} \varphi(x, t) \frac{\partial \Psi}{\partial \gamma} \mathrm{d} S-\int_{\Omega} u(x, t)\left(\lambda_{0} \Psi(x)\right) \mathrm{d} x,
\end{aligned}
$$

where $\mathrm{d} S$ is the surface element on $\partial \Omega$.

From condition (H2), we can easily obtain

$$
\begin{gathered}
\int_{\Omega} p(x, t) f(u(x, t)) \Psi(x) \mathrm{d} x \geq C p(t) \int_{\Omega} u(x, t) \Psi(x) \mathrm{d} x \\
\int_{\Omega} q_{j}(x, t) f_{j}\left(u\left(x, t-\sigma_{j}\right)\right) \Psi(x) \mathrm{d} x \geq C_{j} q_{j}(t) \int_{\Omega} u\left(x, t-\sigma_{j}\right) \Psi(x) \mathrm{d} x .
\end{gathered}
$$

The proof is similar to that of Lemma 2.1 and therefore the details are omitted.

Lemma 3.3. Let $u(x, t) \in C^{2}(\Gamma) \cap C^{1}(\bar{\Gamma})$ be a positive solution of the problem (1), (3) in $G$. If we further assume that $f(-u)=-f(u), \quad u \in(0,+\infty)$ and the impulsive differential inequality (19), and

$$
\left[r(t) v^{\prime}(t)\right]^{\prime}+\lambda_{0} a(t) v(t)+C p(t) v(t)+\sum_{j=1}^{n} C_{j} q_{j}(t) v\left(t-\sigma_{j}\right) \leq-Q(t), t \neq t_{k}
$$




$$
\begin{gathered}
a_{k}^{*} \leq \frac{v\left(t_{k}^{+}\right)}{v\left(t_{k}\right)} \leq a_{k} \\
b_{k}^{*} \leq \frac{v^{\prime}\left(t_{k}^{+}\right)}{v^{\prime}\left(t_{k}\right)} \leq b_{k}, \quad k=1,2, \cdots
\end{gathered}
$$

have no eventually positive solution, then each nonzero solution of the problem (1), (3) is oscillatory in the domain $G$.

Proof. The proof is similar to Lemma 2.3, and hence the details are omitted.

Futhermore, if we set $\varphi \equiv 0$, then we have the following lemma.

Lemma 3.4. Let $u(x, t) \in C^{2}(\Gamma) \cap C^{1}(\bar{\Gamma})$ be a positive solution of the problem (1), (3) in $G$. If we further assume that $f(-u)=-f(u), \quad u \in(0,+\infty)$ and the impulsive differential inequality (19), and

$$
\begin{gathered}
{\left[r(t) v^{\prime}(t)\right]^{\prime}+\lambda_{0} a(t) v(t)+C p(t) v(t)+\sum_{j=1}^{n} C_{j} q_{j}(t) v\left(t-\sigma_{j}\right) \leq 0, t \neq t_{k}} \\
a_{k}^{*} \leq \frac{v\left(t_{k}^{+}\right)}{v\left(t_{k}\right)} \leq a_{k} \\
b_{k}^{*} \leq \frac{v^{\prime}\left(t_{k}^{+}\right)}{v^{\prime}\left(t_{k}\right)} \leq b_{k}, \quad k=1,2, \cdots
\end{gathered}
$$

has no eventually positive solution, then each nonzero solution of the problem (1), satisfying the boundary condition

$$
u=0,(x, t) \in \partial \Omega \times \mathbb{R}^{+}, t \neq t_{k}
$$

is oscillatory in the domain $G$.

Proof. The proof is similar to Lemma 2.4, and hence the details are omitted.

Using the above lemmas, we prove the following oscillation result.

Theorem 3.1. If condition (14) and the following condition

$$
\lim _{t \rightarrow+\infty} \int_{t_{0}}^{t} \prod_{t_{0}<t_{k}<s} \frac{a_{k}^{*}}{b_{k}} r\left(t_{k}\right) F(s) \mathrm{d} s=+\infty,
$$

hold, where

$$
F(t)=\frac{\lambda_{0} a(t)+C p(t)}{r(t)}
$$

then every solution of the problem (1), (3) oscillates in G.

Proof. Let $u(x, t)$ be a nonoscillatory solution of (1), (3). Without loss of generality, we can assume that there exists $T>0, t_{0} \geq T$, such that $u(x, t)>0, u\left(x, t-\sigma_{j}\right)>0, \quad j=1,2, \cdots, n$ for $(x, t) \in \Omega \times\left[t_{0}, \infty\right)$.

From Lemma 3.4, we know that $v(t)$ is a positive solution of (25)-(27). Thus from Lemma 2.6, we can find that $v^{\prime}(t) \geq 0$ for $t \geq t_{0}$.

For $t \geq t_{0}, \quad t \neq t_{k}, \quad k=1,2, \cdots$, define

$$
w(t)=r(t) \frac{v^{\prime}(t)}{v(t)}, \quad t \geq t_{0} .
$$

Then we have $w(t)>0, \quad t \geq t_{0}, \quad r(t) v^{\prime}(t)-w(t) v(t)=0$. We may assume that $v\left(t_{0}\right)=1$, thus we have that for $t \geq t_{0}$

$$
v(t)=\exp \left(\int_{t_{0}}^{t} w(s) \mathrm{d} s\right)
$$




$$
\begin{aligned}
v^{\prime}(t) & =w(t) \exp \left(\int_{t_{0}}^{t} w(s) \mathrm{d} s\right), \\
v^{\prime \prime}(t) & =w^{2}(t) \exp \left(\int_{t_{0}}^{t} w(s) \mathrm{d} s\right) \\
& +w^{\prime}(t) \exp \left(\int_{t_{0}}^{t} w(s) \mathrm{d} s\right) .
\end{aligned}
$$

We substitute (29)-(31) into (25) and can obtain the following inequality,

$$
\begin{aligned}
& r(t)\left[w^{2}(t) \exp \left(\int_{t_{0}}^{t} w(s) \mathrm{d} s\right)+w^{\prime}(t) \exp \left(\int_{t_{0}}^{t} w(s) \mathrm{d} s\right)\right] \\
& +\lambda_{0} a(t) \exp \left(\int_{t_{0}}^{t} w(s) \mathrm{d} s\right)+C p(t) \exp \left(\int_{t_{0}}^{t} w(s) \mathrm{d} s\right) \leq 0,
\end{aligned}
$$

then we have

$$
r(t) w^{\prime}(t)+\lambda_{0} a(t)+C p(t) \leq 0, t \neq t_{k}
$$

From (26)-(27), we can obtain

$$
\begin{aligned}
w\left(t_{k}^{+}\right) & =r\left(t_{k}^{+}\right) \frac{v^{\prime}\left(t_{k}^{+}\right)}{v\left(t_{k}^{+}\right)} \leq r\left(t_{k}^{+}\right) \frac{b_{k} v^{\prime}\left(t_{k}\right)}{a_{k}^{*} v\left(t_{k}\right)} \\
& =r\left(t_{k}\right) \frac{b_{k}}{a_{k}^{*}} w\left(t_{k}\right), \quad k=1,2, \cdots
\end{aligned}
$$

It follows that

$$
\begin{gathered}
r(t) w^{\prime}(t) \leq-\lambda_{0} a(t)-C p(t), \quad t \neq t_{k} . \\
w\left(t_{k}^{*}\right) \leq r\left(t_{k}\right) \frac{b_{k}}{a_{k}^{*}} w\left(t_{k}\right), \quad t=t_{k} .
\end{gathered}
$$

Let

$$
-F(t)=\frac{-\lambda_{0} a(t)-C p(t)}{r(t)} .
$$

Then according to Lemma 2.5, we have

$$
\begin{aligned}
w(t) & \leq w\left(t_{0}\right) \prod_{t_{0}<t_{k}<t} r\left(t_{k}\right) \frac{b_{k}}{a_{k}^{*}}+\int_{t_{0}}^{t} \prod_{s<t_{k}<t} r\left(t_{k}\right) \frac{b_{k}}{a_{k}^{*}} F(s) \mathrm{d} s \\
& =\prod_{t_{0}<t_{k}<t} \frac{b_{k}}{a_{k}^{*}}\left[w\left(t_{0}\right) r\left(t_{k}\right)-\int_{t_{0}}^{t} \prod_{t_{0}<t_{k}<s} r\left(t_{k}\right) \frac{a_{k}^{*}}{b_{k}} F(s) \mathrm{d} s\right]<0 .
\end{aligned}
$$

Since $w(t) \geq 0$, the last inequality contradicts (28). This completes the proof.

Theorem 3.2. If condition (14) and the following condition

$$
\lim _{t \rightarrow+\infty} \int_{t_{t_{0}}}^{t} \prod_{t_{0}<t_{k}<s} \frac{a_{k}^{*}}{b_{k}} r\left(t_{k}\right) C_{j_{0}} q_{j_{0}} \mathrm{~d} s=+\infty,
$$

hold for some $q_{j_{0}}$, then every solution of the problem (1), (3) oscillates in $G$.

Proof. The proof is obvious and hence the details are omitted.

\section{Examples}

In this section, we present some examples to illustrate the main results.

Example 4.1. Consider the impulsive differential equation 


$$
\begin{aligned}
& \frac{\partial}{\partial t}\left((t+\pi)^{2} \frac{\partial}{\partial t}(u(x, t))\right)=(t+\pi)^{2} \sin ^{2}(t) \Delta u(x, t)-(t+\pi)^{2} \cos ^{2}(t) u(x, t) \\
& -2(t+\pi) \sin ^{2}(t) u\left(x, t-\frac{\pi}{2}\right)-(t+\pi) \sin (2 t) \cos (t) \sin (x), t>1, t \neq t_{k}, k=1,2,3, \cdots \\
& u\left(x, t_{k}^{+}\right)=\frac{k+1}{k} u\left(x, t_{k}\right) \\
& u_{t}\left(x, t_{k}^{+}\right)=u_{t}\left(x, t_{k}\right), k=1,2, \cdots
\end{aligned}
$$

and the boundary condition

$$
u(0, t)=u(\pi, t)=0, \quad t \geq 0, \quad t \neq t_{k}, k=1,2, \cdots
$$

Here $\Omega=(0, \pi), a_{k}=a_{k}^{*}=\frac{k+1}{k}, b_{k}=b_{k}^{*}=1, k=1,2, \cdots, r(t)=(t+\pi)^{2}, a(t)=(t+\pi)^{2} \sin ^{2}(t)$, $p(t)=(t+\pi)^{2} \cos ^{2}(t), \quad q_{1}(t)=2(t+\pi) \sin ^{2}(t), \sigma_{1}=\frac{\pi}{2}, f(u)=u, f_{1}(u)=u$, and taking $\left\{t_{k}\right\}_{1}^{+\infty}=\left\{2^{k}\right\}_{1}^{+\infty}$.

Moreover

$$
\begin{aligned}
& \lim _{t \rightarrow+\infty} \int_{t_{t_{0}}<t_{k}<s}^{t} \frac{b_{k}^{*}}{a_{k}} \mathrm{~d} s=\int_{1}^{+\infty} \prod_{1<t_{k}<s} \frac{k}{k+1} \mathrm{~d} s \\
& =\int_{1}^{t_{1}} \prod_{1<t_{k}<s} \frac{k}{k+1} \mathrm{~d} s+\int_{t_{1}^{+}}^{t_{2}} \prod_{1<t_{k}<s} \frac{k}{k+1} \mathrm{~d} s+\int_{t_{2}^{+}}^{t_{1}} \prod_{1<t_{k}<s} \frac{k}{k+1} \mathrm{~d} s+\int_{t_{3}^{+}}^{t_{4}} \prod_{1<t_{k}<s} \frac{k}{k+1} \mathrm{~d} s+\cdots \\
& =1+\frac{1}{2} \times 2+\frac{1}{2} \times \frac{2}{3} \times 2^{2}+\frac{1}{2} \times \frac{2}{3} \times \frac{3}{4} \times 2^{3}+\cdots=\sum_{n=0}^{+\infty} \frac{2^{n}}{n+1}=+\infty
\end{aligned}
$$

so (14) holds. We take $\lambda_{0}=1, C=C_{1}=1, \delta=\max \left\{\sigma_{1}\right\}=\frac{\pi}{2}, w\left(t_{1}\right)=\frac{1}{t+\pi}$, then

$$
F(t)=\frac{(t+\pi)^{2}+(t+\pi)^{2} \cos ^{2}(t)}{(t+\pi)^{2}}=1+\cos ^{2}(t)
$$

thus

$$
\begin{aligned}
\lim _{t \rightarrow+\infty} \int_{1}^{t} \prod_{1<t_{k}<s} \frac{a_{k}^{*}}{b_{k}} r\left(t_{k}\right) F(s) \mathrm{d} s & =\lim _{t \rightarrow+\infty} \int_{1}^{t} \prod_{1<t_{k}<s} \frac{k+1}{k}\left(2^{k}+\pi\right)^{2}\left(1+\cos ^{2}(s)\right) \mathrm{d} s \\
& \geq \lim _{t \rightarrow+\infty} \int_{1}^{t}\left(1+\cos ^{2} s\right) \mathrm{d} s=+\infty .
\end{aligned}
$$

Hence (28) holds. Therefore all conditions of Theorem 3.1 are satisfied. Hence every solution of the problem (33), (34) oscillates in $(0, \pi) \times[0, \infty)$. In fact $u(x, t)=\sin x \cos t$ is one such solution of the problem (33) and (34).

Example 4.2. Consider the impulsive differential equation

$$
\begin{aligned}
& \frac{\partial}{\partial t}\left((t+\pi)^{2} \frac{\partial}{\partial t}(u(x, t))\right)=(t+\pi)^{2} \sin ^{2}(t) \Delta u(x, t)-(t+\pi)^{2} \cos ^{2}(t) u(x, t) \\
& -2(t+\pi) \sin ^{2}(t) u\left(x, t-\frac{5 \pi}{2}\right)+2(t+\pi) \cos ^{3}(t) \cos (x), t>1, t \neq t_{k}, k=1,2,3, \cdots \\
& u\left(x, t_{k}^{+}\right)=\frac{k+1}{k} u\left(x, t_{k}\right) \\
& u_{t}\left(x, t_{k}^{+}\right)=u_{t}\left(x, t_{k}\right), k=1,2, \cdots
\end{aligned}
$$

and the boundary condition 


$$
u_{x}(0, t)=u_{x}(\pi, t)=0, t \geq 0, t \neq t_{k}, k=1,2, \cdots
$$

Here $\Omega=(0, \pi), a_{k}=a_{k}^{*}=\frac{k+1}{k}, b_{k}=b_{k}^{*}=1, k=1,2, \cdots \cdot r(t)=(t+\pi)^{2}, a(t)=(t+\pi)^{2} \sin ^{2}(t)$, $p(t)=(t+\pi)^{2} \cos ^{2}(t), q_{1}(t)=2(t+\pi) \sin ^{2}(t), \sigma_{1}=\frac{5 \pi}{2}, h(u)=0, f(u)=u, f_{1}(u)=u$, and taking $\left\{t_{k}\right\}_{1}^{+\infty}=\left\{2^{k}\right\}_{1}^{+\infty}$. It is easy to check that the conditions of Theorem 2.1 are satisfied. Therefore, every solution of the problem (35), (36) oscillates in $(0, \pi) \times[0, \infty)$. In fact $u(x, t)=\sin t \cos x$ is one such solution of the problem (35) and (36).

\section{Acknowledgements}

The authors thank Prof. E. Thandapani for his support to complete the paper. Also the authors express their sincere thanks to the referee for valuable suggestions.

\section{References}

[1] Wu, J.H. (1996) Theory of Partial Functional Differential Equations and Applications. New York, Springer. http://dx.doi.org/10.1007/978-1-4612-4050-1

[2] Liu, A.P. (1996) Oscillations of Certain Hyperbolic Delay Differential Equations with Damping Term. Mathematica Applicate, 9, 321-324.

[3] Liu, A.P., Xiao, L. and Liu, T. (2002) Oscillations of the Solutions of Hyperbolic Partial Functional Differential Equations of Neutral Type. Acta Analysis Functionalis Applicate, 4, 69-74.

[4] He, M.X. and Liu, A.P. (2003) Oscillation of Hyperbolic Partial Differential Equations. Applied Mathematics and Computation, 142, 205-224. http://dx.doi.org/10.1016/S0096-3003(02)00295-3

[5] Shoukaku, Y. (2011) Oscillation of Solutions for Forced Nonlinear Neutral Hyperbolic Equations with Functional Arguments. Electronic Journal of Differential Equations, 2011, 1-16.

[6] Shoukaku, Y. and Yoshida, N. (2010) Oscillation of Nonlinear Hyperbolic Equations with Functional Arguments via Riccati Method. Applied Mathematics and Computation, 217, 143-151. http://dx.doi.org/10.1016/j.amc.2010.05.030

[7] Yoshida, N. (2008) Oscillation Theory of Partial Differential Equations. World Scientific, Singapore. http://dx.doi.org/10.1142/7046

[8] Erbe, L., Freedman, H., Liu, X.Z. and Wu, J.H. (1991) Comparison Principles for Impulsive Parabolic Equations with Application to Models of Single Species Growth. The Journal of the Australian Mathematical Society. Series B. Applied Mathematics, 32, 382-400. http://dx.doi.org/10.1017/S033427000000850X

[9] Bainov, D.D. and Simeonov, P.S. (1989) Systems with Impulse Effect: Stability Theory and Applications. Ellis Horwood, Chichester.

[10] Bainov, D.D. and Simeonov, P.S. (1993) Impulsive Differential Equations: Periodic Solutions and Applications. Longman, Harlow.

[11] Chan, C. and Ke, L. (1994) Remarks on Impulsive Quenching Problems. Proceedings of Dynamics Systems and Applications, 1, 59-62.

[12] Zhang, L.Q. (2000) Oscillation Criteria for Hyperbolic Partial Differential Equations with Fixed Moments of Impulse Effects. Acta Mathematica Sinica, 43, 17-26.

[13] Mil'man, V.D. and Myshkis, A.D. (1960) On the Stability of Motion in the Presence of Impulses. Siberian Mathematical Journal, 1, 233-237.

[14] Bainov, D.D., Kamont, Z. and Minchev, E. (1996) Monotone Iterative Methods for Impulsive Hyperbolic Differential Functional Equations. Journal of Computational and Applied Mathematics, 70, 329-347. http://dx.doi.org/10.1016/0377-0427(95)00209-X

[15] Cui, B.T., Han, M.A. and Yang, H.Z. (2005) Some Sufficient Conditions for Oscillation of Impulsive Delay Hyperbolic Systems with Robin Boundary Conditions. Journal of Computational and Applied Mathematics, 180, 365-375. http://dx.doi.org/10.1016/j.cam.2004.11.006

[16] Cui, B.T., Liu, Y.Q. and Deng, F.Q. (2003) Some Oscillation Problems for Impulsive Hyperbolic Differential Systems with Several Delays. Applied Mathematics and Computation, 146, 667-679. 
http://dx.doi.org/10.1016/S0096-3003(02)00611-2

[17] Deng, L.H. and Ge, W.G. (2001) Oscillation Criteria of Solutions for Impulsive Delay Parabolic Equations. Acta Mathematica Sinica, 44, 501-506.

[18] Yang, J.C., Liu, A.P. and Liu, G.J. (2013) Oscillation of Solutions to Neutral Nonlinear Impulsive Hyperbolic Equations with Several Delays. Electronic Journal of Differential Equations, 2013, 1-10.

[19] Fu, X.L. and Shiau, L.J. (2013) Oscillation Criteria for Impulsive Parabolic Boundary Value Problem with Delay. Applied Mathematics and Computation, 153, 587-599.

[20] Ye, Q.X. and Li, Z.Y. (1990) Introduction to Reaction Diffusion Equations. Science Press, Beijing.

[21] Lakshmikantham, V., Bainov, D.D. and Simeonov, P.S. (1989) Theory of Impulsive Differential Equations. World Scientific, Singapore. http://dx.doi.org/10.1142/0906

[22] Luo, J.W. (2002) Oscillation of Hyperbolic Partial Differential Equations with Impulses. Applied Mathematics and Computation, 133, 309-318. http://dx.doi.org/10.1016/S0096-3003(01)00217-X 